\title{
Existence of periodic orbits for a strongly non-linear non-ideal problem
}

\author{
Márcio José Horta Dantas ${ }^{1}$ \\ Faculdade de Matemática, UFU, Uberlândia, MG
}

\begin{abstract}
In this paper the existence of periodic orbits for a strongly non linear problem is rigorously proved. The non perturbed part of the system involves a cubic polynomial. In order to approach such problem is necessary to use elliptic functions. For non ideal problems this the first time that such kind of result is obtained.
\end{abstract}

Key words. Periodic Solutions, Regular Perturbation Theory, Poincarè Method, Elliptic Functions

\section{Introduction}

In the literature on non-ideal problems, see for example [1], only weakly non linear problems are approached. By using a line of attack similar to that one used in [4] a rigorous result on existence of periodic orbits for a strongly non linear non ideal problem is obtained. This is in fact only the first step of a research program which aim is to study the Sommerfeld Effect for strongly non linear problems. A rigorous approach for the Sommerfeld Effect in weakly non linear non ideal problems was performed in [3].

In Section 2 a model of the Centrifugal Vibrator is given and some scales are introduced which lead to a non linear problem. In Section 3 by using Regular Perturbation Theory and Poincarè Method the existence result is obtained.

\section{The Centrifugal Vibrator}

We consider a mechanical system excited by a $D C$ motor, with limited supply power, which base is supported on a spring. Besides, the $D C$ motor rotates a small mass $m$, Figure 1. This mechanism has the main properties of a machine known as centrifugal vibrator.

The equations of motion of this system were obtained before by [8], see page 38 . The mathematical model is given by the following system:

$$
\left\{\begin{aligned}
m_{1} \ddot{x}+\beta \dot{x}+c x+d x^{3} & =m r \dot{\varphi}^{2} \cos \varphi+m r \ddot{\varphi} \sin \varphi \\
I \ddot{\varphi} & =M(\dot{\varphi})+m r \ddot{x} \sin \varphi+m g r \sin \varphi
\end{aligned}\right.
$$

\footnotetext{
${ }^{1}$ marcio@ufu.br
} 


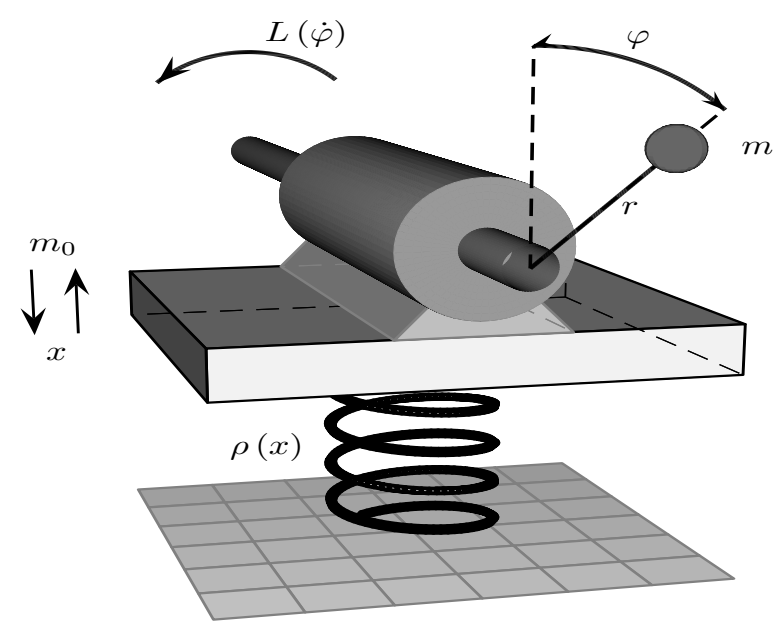

Figure 1: Centrifugal Vibrator

where $m_{1}=m_{0}+m$ and $m_{0}$ denote the mass of the $D C$ motor. The resistance of the oscillatory motion is a linear force $\beta x$. The constant $c$ is the stiffness of the spring. And $d$ is the elasticity coefficient that describes how much the behaviour of the spring moves away from the linear case.

For the remainder of this paper, we consider all constants that appear in equation (1) strictly positive. We will denote by $r$ the distance between the mass $m$ and the axis of rotation of the $D C$ motor. $J$ and $m r^{2}$ are the moment of inertia of the rotating parts of the $D C$ motor and the moment of inertia of the rotating mass $m$, respectively. Therefore, the total moment of inertia of the system is given by $I=J+m r^{2}$. Furthermore, $g$ denotes the acceleration of gravity. Note that the function $M(\cdot)$ is the difference between the driving torque of the source of energy (motor) and the resistive torque applied to the rotor. Such function $M(\cdot)$ is obtained from experiments.

Remark 2.1. In some cases, see for example [6, eq.4], one can take $M(\dot{\varphi})=a-b \dot{\varphi}$ where $a, b>0$. This is important because satisfies a hypothesis of our existence result.

We can rewrite the equations of motion (1) as a system of first order. Taking

$$
\begin{aligned}
& \omega^{2}=\frac{c}{m_{1}}, \quad a_{2}=-\frac{\beta}{m_{1}}, a_{3}=\frac{d}{m_{1}}, a_{4}=\frac{m r}{m_{1}}, a_{5}=\frac{m r}{I}, \\
& a_{6}=\frac{m g r}{I}, M_{1}(\dot{\varphi})=\frac{M(\dot{\varphi})}{I} .
\end{aligned}
$$

Now, let us introduce a small parameter $\varepsilon$ in the above parameters. Let us replace the parameters $a_{i}, i \neq 3$ and $\omega$ by $\varepsilon a_{i}, i \neq 3$ and $\varepsilon \omega$ respectively. Moreover, let us substitute $M_{1}(\dot{\varphi})$ by $\varepsilon M_{1}(\dot{\varphi})$. Hence from (1) one obtains

$$
\left\{\begin{array}{rrr}
\ddot{x}+\varepsilon^{2} \omega^{2} x & = & -a_{3} x^{3}+\varepsilon\left(a_{4} \dot{\varphi}^{2} \cos \varphi+a_{4} \ddot{\varphi} \sin \varphi+a_{2} \dot{x}\right), \\
\ddot{\varphi} & = & \varepsilon\left(M_{1}(\dot{\varphi})+a_{5} \ddot{x} \sin \varphi+a_{6} \sin \varphi\right)
\end{array} .\right.
$$

By making $x_{1}=x, x_{2}=\dot{x}, x_{3}=\varphi, x_{4}=\dot{\varphi}$ in (2) it is obtained after a 
long, but straightforward, computation that

$$
\left(\begin{array}{l}
\dot{x}_{1} \\
\dot{x}_{2} \\
\dot{x}_{3} \\
\dot{x}_{4}
\end{array}\right)=\left(\begin{array}{l}
x_{2} \\
-a_{3} x_{1}^{3}+\varepsilon\left(a_{2} x_{2}+a_{4} x_{4}^{2} \cos x_{3}\right) \\
x_{4} \\
\varepsilon\left(M_{1}\left(x_{4}\right)+a_{6} \sin x_{3}\right)
\end{array}\right)+O\left(\varepsilon^{2}\right) .
$$

\subsection{A Change of Variables and Reduction}

From now on jacobian elliptic functions will be used in the next steps. Basic references are [5] and [9].

Remark 2.2. The jacobian cosine with modulus $1 / \sqrt{2}$ is denoted by $\mathrm{cn}(t, 1 / \sqrt{2})$ and its period by $k_{0}$. Note that $c n^{\prime \prime}(t, 1 / \sqrt{2})=-c n^{3}(t, 1 / \sqrt{2})$.

By taking $x_{1}=C c n\left(D, \frac{1}{\sqrt{2}}\right), x_{2}=\sqrt{a_{3}} C^{2} c n^{\prime}\left(D, \frac{1}{\sqrt{2}}\right)$ in (3) we get

$$
\begin{aligned}
& \left(\begin{array}{c}
\dot{C} \\
\dot{D} \\
\dot{x}_{3} \\
\dot{x}_{4}
\end{array}\right)=\left(\begin{array}{c}
0 \\
\sqrt{a_{3}} C \\
x_{4} \\
0
\end{array}\right) \\
& +\epsilon\left(\begin{array}{c}
\frac{a_{2} C}{2}-\frac{a_{2} C c n^{4}\left(D, \frac{1}{\sqrt{2}}\right)}{2}+\frac{a_{4} x_{4}^{2} \cos \left(x_{3}\right) c n^{\prime}\left(D, \frac{1}{\sqrt{2}}\right)}{\sqrt{a_{3}} C} \\
-a_{2} c n\left(D, \frac{1}{\sqrt{2}}\right) c n^{\prime}\left(D, \frac{1}{\sqrt{2}}\right)-\frac{a_{4} x_{4}^{2} \cos \left(x_{3}\right) c n\left(D, \frac{1}{\sqrt{2}}\right)}{\sqrt{a_{3}} C^{2}} \\
0 \\
M_{1}\left(x_{4}\right)+a_{6} \sin \left(x_{3}\right)
\end{array}\right) \\
& +O\left(\epsilon^{2}\right) \text {. }
\end{aligned}
$$

Using the usual reduction process in (4) one has

$$
\begin{aligned}
& \left(\begin{array}{c}
\frac{d C}{d x_{3}} \\
\frac{d D}{d x_{3}} \\
\frac{d x_{4}}{d x_{3}}
\end{array}\right)=\left(\begin{array}{c}
0 \\
\frac{\sqrt{a_{3}} C}{x_{4}} \\
0
\end{array}\right) \\
& +\epsilon\left(\begin{array}{c}
\frac{\left(2 \sqrt{a_{3}} a_{4} x_{4}^{2} \cos \left(x_{3}\right) c n^{\prime}\left(D, \frac{1}{\sqrt{2}}\right)+a_{2} C^{2}\left(a_{3}-a_{3} c n^{4}\left(D, \frac{1}{\sqrt{2}}\right)\right)\right)}{2 a_{3} x_{4} C} \\
-\frac{\left(a_{2} a_{3} C^{2} c n\left(D, \frac{1}{\sqrt{2}}\right) c n^{\prime}\left(D, \frac{1}{\sqrt{2}}\right)+\sqrt{a_{3}} a_{4} x_{4}^{2} \cos \left(x_{3}\right) c n\left(D, \frac{1}{\sqrt{2}}\right)\right)}{a_{3} x_{4} C^{2}} \\
\frac{\left(M_{1}\left(x_{4}\right)+a_{6} \sin \left(x_{3}\right)\right)}{x_{4}}
\end{array}\right) \\
& +O\left(\epsilon^{2}\right) .
\end{aligned}
$$


Consider the following change of variables $D\left(x_{3}\right)=D_{1}\left(x_{3}\right)-\frac{k_{0} x_{3}}{2 \pi}$. By using this change of variables into (5) one obtains

$$
\begin{aligned}
& \left(\begin{array}{c}
\frac{d C}{d x_{3}} \\
\frac{d D_{1}}{d x_{3}} \\
\frac{d x_{4}}{d x_{3}}
\end{array}\right)=\left(\begin{array}{c}
0 \\
\frac{2 \pi \sqrt{a_{3}} C+k_{0} x_{4}}{2 \pi x_{4}} \\
0
\end{array}\right) \\
& +\epsilon\left(\begin{array}{c}
\frac{\left(2 \sqrt{a_{3}} a_{4} x_{4}^{2} \cos \left(x_{3}\right) c n^{\prime}\left(D_{1}-\frac{k_{0} x_{3}}{2 \pi}, \frac{1}{\sqrt{2}}\right)+a_{2} C^{2}\left(a_{3}-a_{3} c n^{4}\left(D_{1}-\frac{k_{0} x_{3}}{2 \pi}, \frac{1}{\sqrt{2}}\right)\right)\right)}{2 a_{3} x_{4} C} \\
-\frac{\left(a_{2} a_{3} C^{2} c n\left(D_{1}-\frac{k_{0} x_{3}}{2 \pi}, \frac{1}{\sqrt{2}}\right) c n^{\prime}\left(D_{1}-\frac{k_{0} x_{3}}{2 \pi}, \frac{1}{\sqrt{2}}\right)+\sqrt{a_{3}} a_{4} x_{4}{ }^{2} \cos \left(x_{3}\right) c n\left(D, \frac{1}{\sqrt{2}}\right)\right)}{a_{3} x_{4} C^{2}} \\
\frac{\left(M_{1}\left(x_{4}\right)+a_{6} \sin \left(x_{3}\right)\right)}{x_{4}}
\end{array}\right) \\
& +O\left(\epsilon^{2}\right) .
\end{aligned}
$$

Observe that (6) is $2 \pi$ periodic system in the "time" $x_{3}$. The main aim of this paper is to prove the existence of $2 \pi$ periodic orbits for this system.

\section{Searching for Periodic Orbits: A Perturbation Approach}

In this section the Regular Perturbation Theory and the Poincaré Method are used in order to obtain our existence result. A good reference for both is [2].

Taking into account the Remark 2.1, let us assume the following condition on $M_{1}$, There is $s_{0}>0$ such that

$$
M_{1}\left(s_{0}\right)=0 \text { and } M_{1}^{\prime}\left(s_{0}\right) \neq 0 .
$$

From now on, the variable $x_{3}$ will be replaced by $s$ in (6). In this system take the following initial conditions $\left(C(0), D_{1}(0), x_{4}(0)\right)=\left(a, b, c_{0}\right)+\epsilon(0,0, c)$ where $c_{0}$ is an adequately chosen parameter. Let $\Phi$ be the flow of (6). It is well known, see [7, Th. 3.3, pg.21], that $\Phi$ is a $C^{\infty}$ mapping of the initial conditions as well as of the parameters. Hence from Taylor's formula

$$
\Phi\left(s, a, b, c_{0}+\epsilon c, \epsilon\right)=\Psi_{0}\left(s, a, b, c_{0}\right)+\Psi_{1}(s, a, b, c) \epsilon+O\left(\epsilon^{2}\right)
$$

where $\Psi_{i}, i=0,1$ and the remainder $O\left(\epsilon^{2}\right)$ are $C^{\infty}$ mappings. One has that $\Psi_{0}\left(s, a, b, c_{0}\right)=$ $\left(C_{0}(s), D_{10}(s), x_{40}(s)\right)$ satisfies the following system

$$
C_{0}^{\prime}(s)=0, D_{10}^{\prime}(s)=\frac{2 \pi \sqrt{a_{3}} C_{0}(s)+k_{0} x_{40}(s)}{2 \pi x_{40}(s)}, x_{40}^{\prime}(s)=0
$$

with initial conditions given by $C_{0}(0)=a, D_{10}(0)=b, x_{40}(0)=c_{0}$. By taking $c_{0}=$ $-\frac{2 \pi a \sqrt{a_{3}}}{k_{0}}$ one obtains from the foregoing initial conditions and (9) that

$$
C_{0}(s)=a, D_{10}(s)=b, x_{40}(s)=-\frac{2 \pi a \sqrt{a_{3}}}{k_{0}} .
$$


Similarly $\Psi_{1}(s, a, b, c)=\left(C_{1}(s), D_{11}(s), x_{41}(s)\right)$ can be obtained in this way, So

$$
\begin{aligned}
C_{1}^{\prime}(s)= & -\frac{1}{4 \pi k_{0} \sqrt{a_{3}}}\left(8 \pi^{2} \sqrt{a_{3}} a_{4} \cos (s) c n^{\prime}\left(-\frac{k_{0} s-2 \pi b}{2 \pi}, \frac{1}{\sqrt{2}}\right)\right. \\
& \left.-k_{0}^{2} a_{2} c n^{4}\left(-\frac{k_{0} s-2 \pi b}{2 \pi}, \frac{1}{\sqrt{2}}\right)+k_{0}^{2} a_{2}\right) \\
D_{11}^{\prime}(s)= & \frac{1}{4 \pi^{2} k_{0} a a_{3}}\left(\sqrt { a _ { 3 } } \left(2 \pi k_{0}^{2} a_{2} c n\left(-\frac{k_{0} s-2 \pi b}{2 \pi}, \frac{1}{\sqrt{2}}\right)\right.\right. \\
& \left.\times c n^{\prime}\left(-\frac{k_{0} s-2 \pi b}{2 \pi}, \frac{1}{\sqrt{2}}\right)-k_{0}^{3} x_{41}(s)\right) \\
& \left.+8 \pi^{3} a_{3} a_{4} \cos (s) c n\left(-\frac{k_{0} s-2 \pi b}{2 \pi}, \frac{1}{\sqrt{2}}\right)-2 \pi k_{0}^{2} a_{3} C_{1}(s)\right), \\
x_{41}^{\prime}(s)= & -\frac{k_{0} a_{6} \sin (s)+k_{0} M_{1}\left(-\frac{2 \pi a \sqrt{a_{3}}}{k_{0}}\right)}{2 \pi a \sqrt{a_{3}}}
\end{aligned}
$$

with initial conditions given by $C_{1}(0)=0, D_{11}(0)=0, x_{41}(0)=c$. One obtains from these conditions, (11) and (13) that

$$
\begin{aligned}
C_{1}(s)= & -\frac{1}{4 \pi k_{0}^{2} \sqrt{a_{3}}}\left(16 \pi^{3} \sqrt{a_{3}} a_{4} \int_{b-\frac{k_{0} s}{2 \pi}}^{b} c n^{\prime}\left(u, \frac{1}{\sqrt{2}}\right) \cos \left(\frac{2 \pi u}{k_{0}}-\frac{2 \pi b}{k_{0}}\right) d u\right. \\
& \left.-2 \pi k_{0}^{2} a_{2} \int_{b-\frac{k_{0} s}{2 \pi}}^{b} c n\left(u, \frac{1}{\sqrt{2}}\right)^{4} d u+k_{0}^{3} a_{2} s\right)
\end{aligned}
$$

and

$$
x_{41}(s)=\frac{k_{0} a_{6} \cos (s)}{2 \pi \sqrt{a_{3}} a}-\frac{k_{0} M_{1}\left(-\frac{2 \pi \sqrt{a_{3}} a}{k_{0}}\right)}{2 \pi \sqrt{a_{3}} a} s-\frac{k_{0} a_{6}}{2 \pi \sqrt{a_{3}} a}+c .
$$

By substituting (14) and (15) into (12) and after a long computation, one gets

$$
D_{11}(s)=F(s, a, b)-\frac{k_{0}^{2} c}{4 \pi^{2} a \sqrt{a_{3}}} s
$$

where $F(s, a, b)$ has a huge expression and it will be omitted here. Anyway, one has $F(0, a, b)=0$. Hence from $(8),(10)$ one obtains that

$$
\Phi\left(s, a, b, c_{0}+\epsilon c, \epsilon\right)=\left(a, b,-\frac{2 \pi a \sqrt{a_{3}}}{k_{0}}\right)+\epsilon\left(C_{1}(s), D_{11}(s), x_{41}(s)\right)+O\left(\epsilon^{2}\right) .
$$

The system (6) has a $2 \pi$ solution if, and only if,

$$
\Phi\left(2 \pi, a, b, c_{0}+\epsilon c, \epsilon\right)=\Phi\left(0, a, b, c_{0}+\epsilon c, \epsilon\right) .
$$


From (17), one has that (18) reduces itself to

$$
\left(C_{1}(2 \pi), D_{11}(2 \pi), x_{41}(2 \pi)-c\right)+O(\epsilon)=(0,0,0) .
$$

A direct computation from (14) shows that

$$
C_{1}(2 \pi)=\frac{4 \pi^{2} k_{1} a_{4}}{k_{0}^{2}} \sin \left(\frac{2 \pi b}{k_{0}}\right)-\frac{a_{2}\left(k_{2}-k_{0}\right)}{2 \sqrt{a_{3}}}
$$

where

$$
k_{1}=\int_{0}^{k_{0}} c n^{\prime}\left(u, \frac{1}{\sqrt{2}}\right) \sin \left(\frac{2 \pi u}{k_{0}}\right) d u, k_{2}=\int_{0}^{k_{0}} \operatorname{cn}\left(u, \frac{1}{\sqrt{2}}\right)^{4} d u
$$

Then, by using (20), (15), (16) into (19) one has

$$
\begin{aligned}
&\left(\frac{4 \pi^{2} k_{1} a_{4}}{k_{0}^{2}} \sin \left(\frac{2 \pi b}{k_{0}}\right)-\frac{a_{2}\left(k_{2}-k_{0}\right)}{2 \sqrt{a_{3}}}, F(2 \pi, a, b)-\frac{k_{0}^{2} c}{2 \pi a \sqrt{a_{3}}},\right. \\
&\left.-\frac{k_{0} M_{1}\left(-\frac{2 \pi \sqrt{a_{3}} a}{k_{0}}\right)}{\sqrt{a_{3}} a}\right)+O(\epsilon)=(0,0,0) .
\end{aligned}
$$

Let us denote by $\Psi(a, b, c, \epsilon)$ the left-hand side of (21). From the properties of the flow one concludes that $\Psi$ is $C^{\infty}$ mapping. Let us assume

$$
\left|\frac{k_{0}^{2} a_{2}\left(k_{2}-k_{0}\right)}{8 \pi^{2} k_{1} \sqrt{a_{3}} a_{4}}\right|<1
$$

Consider $\bar{a}, \bar{b}, \bar{c}$ such that

$$
M_{1}\left(-\frac{2 \pi \sqrt{a_{3}} \bar{a}}{k_{0}}\right)=0, \sin \left(\frac{2 \pi \bar{b}}{k_{0}}\right)=\frac{k_{0}^{2} a_{2}\left(k_{2}-k_{0}\right)}{8 \pi^{2} k_{1} \sqrt{a_{3}} a_{4}}, \bar{c}=\frac{2 \pi \bar{a} \sqrt{a_{3}} F(2 \pi, \bar{a}, \bar{b})}{k_{0}^{2}} .
$$

Of course due to (7) and (23) one has $-\frac{2 \pi \sqrt{a_{3}} \bar{a}}{k_{0}}=s_{0}$. In view of (22) the above system has at least two solutions. Anyway, one has that $\Psi(\bar{a}, \bar{b}, \bar{c}, 0)=0$. Then

$$
\operatorname{det}\left(\frac{\partial \Psi}{\partial(a, b, c)}(\bar{a}, \bar{b}, \bar{c}, 0)\right)=-\frac{8 \pi^{3} k_{1} a_{4}}{k_{0} \sqrt{a_{3}} \bar{a}^{2}} M_{1}^{\prime}\left(-\frac{2 \pi \sqrt{a_{3}} \bar{a}}{k_{0}}\right) \cos \left(\frac{2 \pi \bar{b}}{k_{0}}\right) .
$$

Because (22) and (7) hold the determinant in (24) is different from zero. Then, it follows from Implicit Function Theorem that there $C^{\infty}$ functions $a(\epsilon), b(\epsilon), c(\epsilon)$ with $a(0)=$ $\bar{a}, b(0)=\bar{b}, c(0)=\bar{c}$ such that

$$
\Psi(a(\epsilon), b(\epsilon), c(\epsilon), \epsilon)=0
$$

for all $\epsilon$ adequately small. From the above construction one concludes that $\Phi\left(s, a(\epsilon), b(\epsilon), c_{0}+\epsilon c(\epsilon), \epsilon\right)$ is $2 \pi$ is a periodic solution of $(6)$ for $\epsilon \ll 1$. 


\section{Acknowledgements}

The author acknowledges the support given by FAPEMIG.

\section{References}

[1] J. M. Balthazar, D. T. Mook, H. I. Weber, R. M. L. R. F.Brasil, A. Fenili, D. Belato, J. L. P. Felix, An overview on non-ideal vibrations, Meccanica, 38,613-621, 2003.

[2] J. Cronin, Ordinary Differential Equations, Chapman \& Hall/CRC, Boca Raton, 2008.

[3] M.J.H. Dantas and J. M. Balthazar, On the existence and stability of periodic orbits in non ideal problems: General results, Journal of Sound and Vibration, 315,10471070, 2008.

[4] M.J.H. Dantas and J. M. Balthazar, On energy transfer between linear and nonlinear oscillators, Z. angew. Math. Phys. (ZAMP), 58,940-958, 2007, .

[5] H.T.Davis, Introduction to Nonlinear Differential and Integral Equations, Dover Publications, Mineola, New York 1962.

[6] J. L. P. Felix, J. M. Balthazar and M. J. H. Dantas. On a Nonideal(MRD) DamperElectro-Mechanical absorber dynamics, International Journal of Bifurcation and Chaos, 21:10, 2871-2882, 2011.

[7] J. Hale, Ordinary Differential Equations, Dover Publications, Mineola, New York 2009.

[8] V. Kononenko. Vibrating Systems with Limited Power Supply, Illife Books, London, 1969.

[9] E.T. Whittaker and G.N.Watson, A course of Modern Analysis, Cambridge University Press, Cambridge, 1973. 